\title{
Performance evaluation of a few- and multimode fiber optic perimeter sensor with selective mode excitation
}

\author{
Rosen Miletiev, ${ }^{1}$ Rumen Arnaudov, ${ }^{1}$ Wojtek Bock, ${ }^{2}$ Tinko Eftimov, ${ }^{* 3}$ and Xiaoyi Bao ${ }^{4}$ \\ ${ }^{I}$ Faculty of Communications and Communication Technologies, Technical University of Sofia, Bulgaria, \\ ${ }^{2}$ Centre de recherche en Photonique, Département d'informatique et d'ingénierie, \\ Université du Québec en Outaouais, Gatineau, Québec, Canada \\ ${ }^{3}$ Faculty of Physics, Plovdiv University, Plovdiv, Bulgaria \\ ${ }^{4}$ Faculty of Physics, University of Ottawa, Ottawa, Canada
}

Received February 9, 2010; accepted March 26, 2010; published March 31, 2010

\begin{abstract}
We present a study of the performance of a simple fiberoptic perimeter snsor based on selective mode excitation in few- and multimode optical fibers. The electronic unit identifies an intrusion on the basis of a pre-defined threshold and number of detected pulses per unit time. The sensor may be controlled using the keypad, a PC or a FSK modem. The statistical properties of the responses are analyzed using Weibull distributions.
\end{abstract}

A fiber-optic perimeter sensor (FOPS) is a security tool using a fiber-optic cable to detect perturbations (fiber bending, torsion or movement). The FOPS detects changes in the irregular intermodal interference speckle pattern arising in a few-mode (FMF) or multimode fiber (MMF) propagating a coherent laser wave. They are caused by door opening, bumping into or climbing onto a fence, unauthorized removal of various articles such as computers, cars, motorcycles, bicycles, etc., to which the fiber must be attached and are interpreted as an intrusion within a security area.

Various methods to detect specklegram changes have been tested such as phase conjugate mirror (PCM) [1] holographic detection [2] induced mode coupling, [3] joint-transform correlator (JTC) [4,5], mean-absolute speckle-intensity variation (MSV) using a CCD camera [6], spatial filtering technique [7] by butt-joining fibers with different core diameters, a reflection-type speckle interferometer combined with spatial mode filtering [8] as well as a sophisticated combination of threshold and frequency filtering based on fast Fourier transform [9].

We extend here the previous study [10] of a hand-held fiber-optic perimeter sensor that uses low-cost conventional components and allows flexibility for a variety of applications. We use selective mode excitation, a number of decision criteria such as a signal threshold and temporal sequence of time differentiated signal pulses to discriminate between slow- and fast-varying signals as well as all-fiber spatial filtering that is polarizationsensitive.

"E-mail: teftimov@uni-plovdiv.bg
The temporal coherence of the laser source $(\lambda=635$ $\mathrm{nm})$ allows for fiber lengths of several hundred meters to be used. Standard communication fibers (SMF-28, fewmode at $635 \mathrm{~nm}$ and $65 / 125 \mu \mathrm{m}$ multimode fiber) are used as sensing fibers in which higher-order modes are selectively excited. Also, additional fibers can be added to the sensing loop to extend its length.

We have tested the repeatability of the sensor's responses to several possible perturbations: pushing $(\mathrm{P})$ a straight fiber against a soft cushion or in the air; rolling (R) a fiber cable upon a surface; a helical twist $(\mathrm{H})$ through an angle $\phi$; loop curvature (C) change; a simulation of opening/closing a door (O) through an angle $\theta$ as shown in Fig. 1.

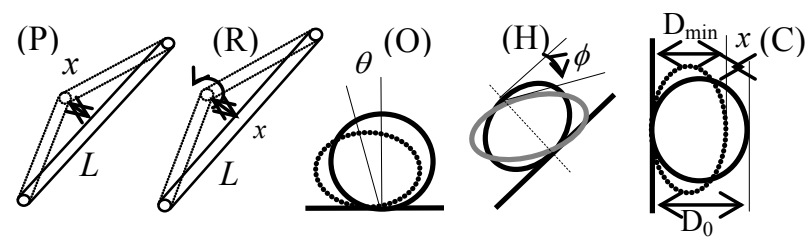

Fig.1 Schematic representations of five tested perturbations.

We have investigated the signals $U(t)$ and the difference $\Delta U(t)=U(t+\Delta t)-U(t)$ proportional to induced changes [10], the delay $\Delta \mathrm{t}$ typically equivalent to $6.66 \mathrm{~ms}$ and. Responses for $\mathrm{P}, \mathrm{S}, \mathrm{R}$ and $\mathrm{O}$ disturbances on $\mathrm{MMF}$ are shown in Fig. 2 to 5. Due to te relaxation of fiber in the $3 \mathrm{~mm}$ furcation tubing, a slow drift of the signal can sometimes be present as seen in Figures 2 and 3 . However, the use of differential signal $\Delta U(t)$ eliminates drift problems. 


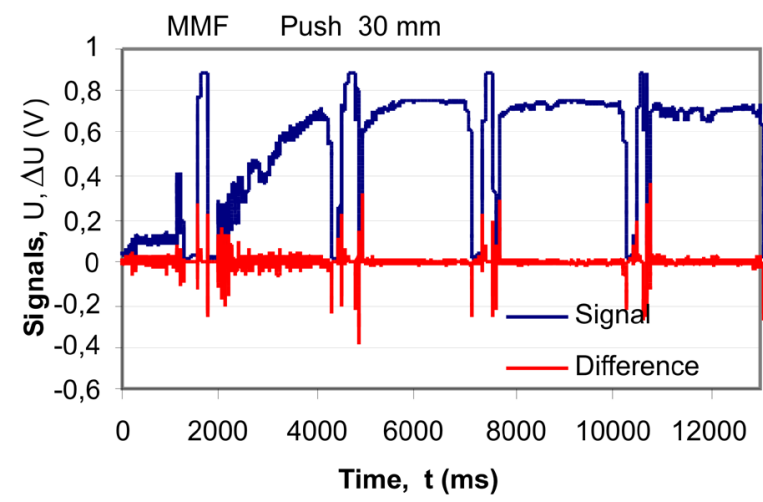

Fig. 2 Responses of four consecutive $3 \mathrm{~mm}$ loop curvature deformations (P) in an MMF.

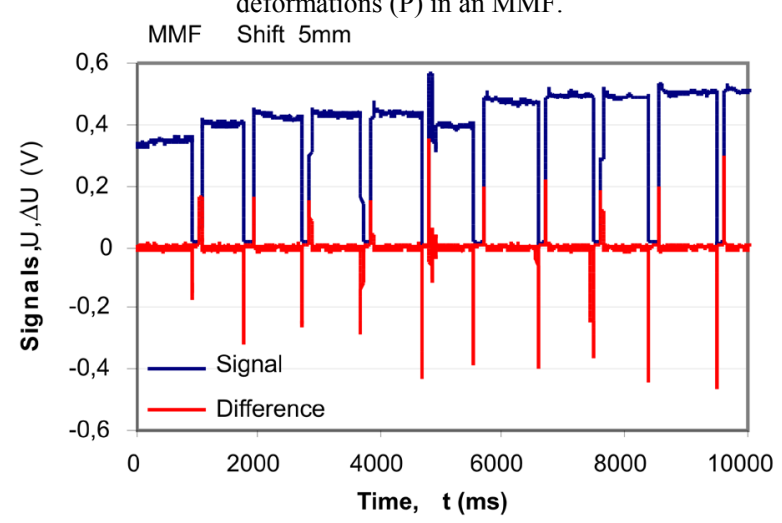

Fig. 3 Responses of consecutive deviation deformations (S) $x=5 \mathrm{~mm}$

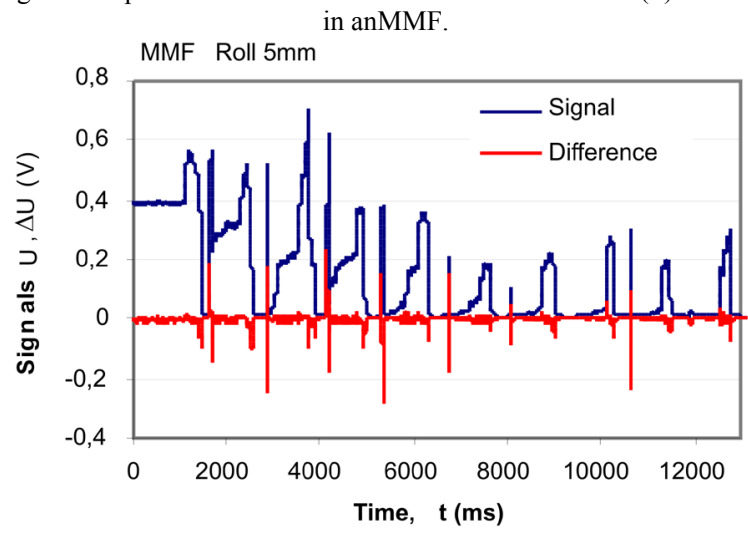

Fig. 4 Responses of consecutive $5 \mathrm{~mm}$ rolling deformations (R) in an MMF.

In all of the above figures we see that the use of a differential signal removes low frequency fluctuations and drifts, and the obtained pulses are usually in the \pm 0.2 $\mathrm{V}$ range. Different types of disturbances show specific responses and, under other equal conditions, the MMF generates more pulses than the FMF. Figures 6 to 9 summarize the observations and present the probabilities for different disturbances to generate a given number of pulses, the threshold being $0.1 \mathrm{~V}$, which is a very high level with respect to typical noises.

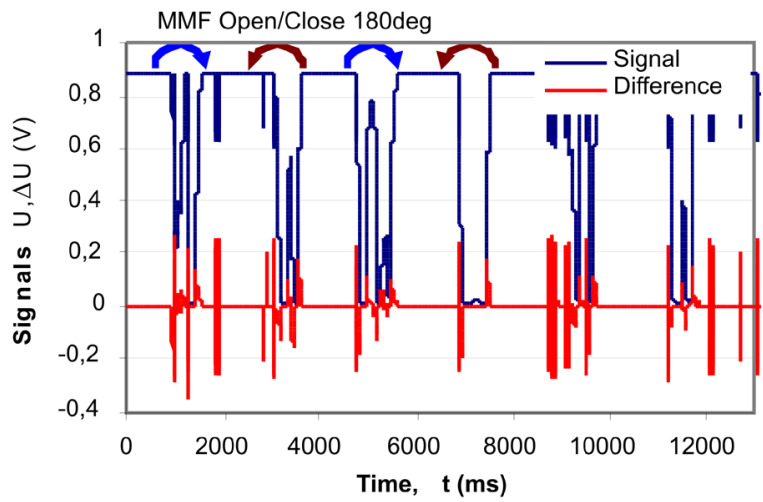

Fig. 5 Responses of consecutive $180 \mathrm{deg}$ open/close (O) deformations in an MMF.

As seen for all types of perturbations, the average number of generated pulses is greater than one, and with the exception of the weak $2 \mathrm{~mm}$ pushing deformation (Fig. 6), the probability to have zero pulses is zero, which is also true for the few-mode fiber.

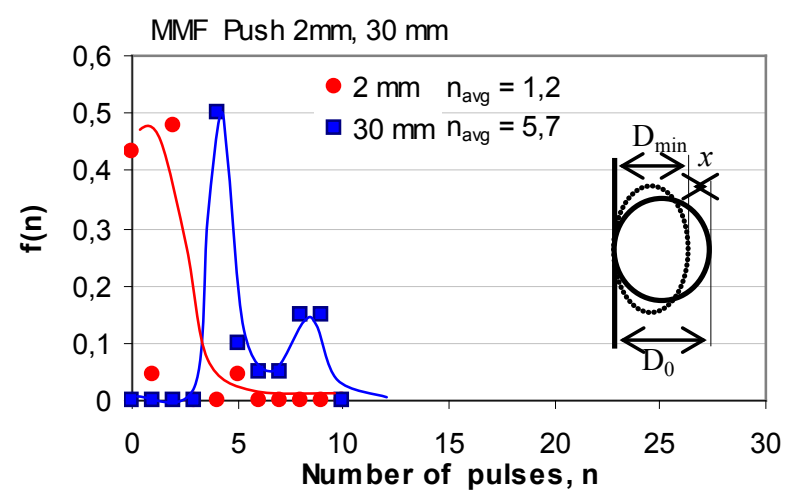

Fig.6 Probability density of pulses in the $\Delta U(t)$ responses of the curvature deformation for $x=2 \mathrm{~mm}$ and $30 \mathrm{~mm}$.

Typically, a second maximum in the distribution tends to appear with the amplitude of perturbation, which is clearly visible in all the figures. The "open/close" disturbance is caused by rotating fiber loops attached on doors. The distribution shows a larger number of pulses for a greater rotation angle. The tests of the developed FOPS show that it can operate with a few-mode and a multimode sensing fiber or combinations of both. We have found that the responses from an MMF produce less scattered data. This, in turn, entails smaller minimum values $x_{\min }$ of the perturbing physical quantity to ensure a given level of detection probability $F_{\min }$. The reason for the greater sensitivity of multimode optical fibers is that the detection unit reads changes in the intermodal interference within a small area of the whole speckle pattern. Since the number of speckles is proportional to the number of modes, more changes will be observed per unit detecting surface. 


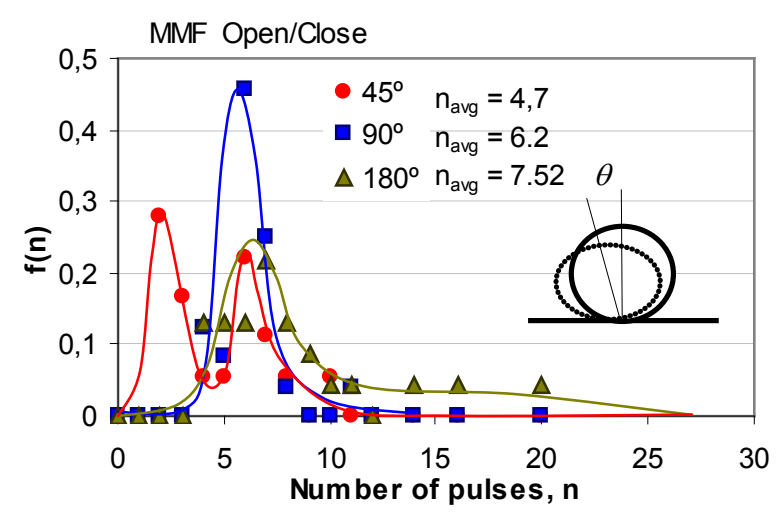

Fig.7 Probability density of pulses in the $\Delta \mathrm{U}(\mathrm{t})$ responses for the "open/close" deformation for $\theta=45^{\circ}, 90^{\circ}$ and $180^{\circ}$.

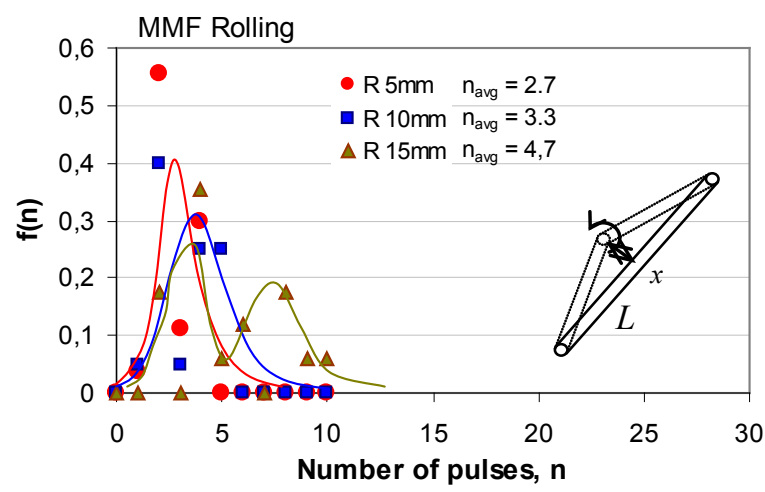

Fig. 8 Probability density of pulses in the $\Delta \mathrm{U}(\mathrm{t})$ responses for a rolling disturbance.

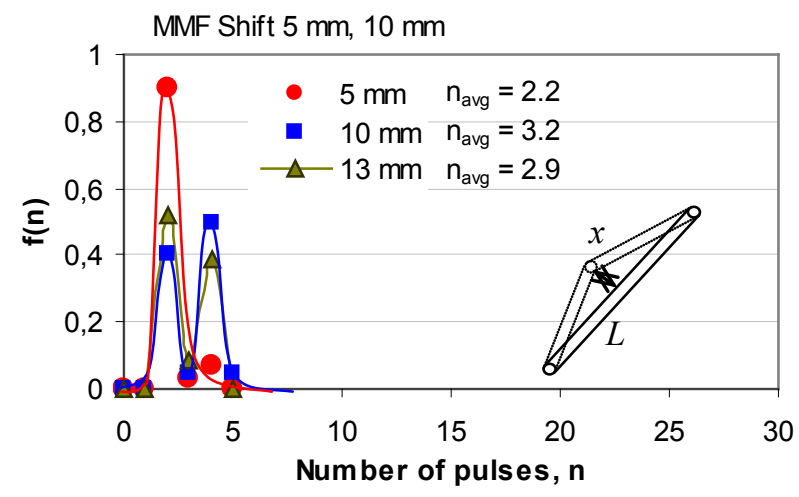

Fig. 9. Probability density of pulses in the $\Delta \mathrm{U}(\mathrm{t})$ responses for a simple deviation of varying amplitude and $x=5 \mathrm{~mm}$ and $10 \mathrm{~mm}$.

For some perturbations such as twists and helical twists and "open/close", the mode pattern rotation of higher-order modes occurs [11] and the higher the mode order, the more changes per unit detecting area. This effect is also polarization-sensitive.

In spite of the greater sensitivity of the sensor operating with multimode fibers, the few-mode regime of operation is very important for developing a simple handheld security sensor that senses the integrity of fiber communication systems in which the fiber is single-mode at $1300 \mathrm{~nm}$ and $1550 \mathrm{~nm}$ but is few-mode at $650 \mathrm{~nm}$. By changing the decision criteria for triggering an alarm, the sensitivity of the sensor may be varied and improved. Most importantly, the decision criteria must be imposed not on the signal $U(t)$ itself, but on the first derivative $\Delta \mathrm{U}(\mathrm{t}) / \mathrm{dt}$ because in this way slow drifts in the signal, caused by temperature variations and fiber relaxation in the tubing are successfully eliminated. This would be analogous to filtering low-frequency noises [9].

In conclusion, we have developed a prototype of a simple, hand-held fiber-optic perimeter sensor which can function with both multimode and few-mode fibers as well as with stacked fibers of the same type and combinations of both. The sensor can be connected to external devices such as computers, cellular phones, etc.

The statistical responses of the sensor to particular disturbances have been studied using Weibull distributions that allow simple evaluation of the minimum level of disturbance needed to have a desired level of probability for an alarm.

The repeatability of pulses under various disturbances has been studied and both the original signal and a differential signal proportional to the first derivative have been considered.

The probability distributions of the number of generated differential pulses have been plotted, from which it follows that the average number of pulses is greater than one.

Support for this work by the NSERC of Canada and by the Canada Research Chairs Program and from the Internal Research Funding under grant F-48 of the Plovdiv University are gratefully acknowledged.

\section{References}

[1] A. Yariv, J. Opt. Soc. Amer., 66, 301(1976)

[2] A. S. Wu, S. Yin and F.T.S. Yu, , Appl. Opt. 30, 4468 (1991).

[3] S. Yin, P. Purwosumarto and F.T.S.Yu, Opt. Commun. 170, 15 (1999)

[4] A. F. T.S. Yu, K.Pan, C. Uang and P.B. Ruffin, Opt. Eng. 32, 2884 (1993).

[5] F. T.S. Yu, K.Pan, D. Zhao and P.B. Ruffin, Appl. Opt. 34, 622 (1995).

[6] K.Pan, C.-M.Uang, F.Cheng and F.T.S. Yu, Appl. Opt. 33, 2095 (1994).

[7] A. Malki, R. Gafsi, L. Michel, M. Labarrère and P. Lecoy, Appl. Opt. 35, 5198 (1996).

[8] J. Park, J. of the Korean Phys. Soc. 50, 529 (2007).

[9] D. Anderson, Fiber SenSys White Paper, http://www.fibersensys.com/index.php?option=com docman\&task $=$ doc view\&gid=56\&tmpl=component\&format $=$ raw\&Itemid $=54$

[10] R.Arnaudov, W. Bock, R.Miletiev, Y. Angelov and T. Eftimov, IMTC 2007, Warsaw, Poland, paper IM-7352(2007).

[11] T.Eftimov and T.Kortenski, Bulg. J. Phys. 14, 456(1987). 